\title{
Broken-helix antiferromagnetic order protecting a crystalline axion insulator phase and exotic surface states in Euln2As2.
}

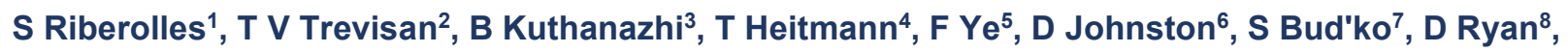 \\ P Canfield ${ }^{9}$, A Kreyssig ${ }^{10}$, A Vishwanath ${ }^{11}$, R McQueeney ${ }^{12}$, L L Wang ${ }^{13}$ \\ ${ }^{1}$ Ames Laboratory ${ }^{2}$ Department of Physics and Astronomy, Ames Laboratory, lowa State \\ University, ${ }^{3}$ Department of Physics and Astronomy, Ames Laboratory, lowa State University, \\ ${ }^{4}$ Missouri Research Reactor, University of Missouri, ${ }^{5}$ No affiliation given, ${ }^{6}$ Department of Physics \\ and Astronomy, Ames Laboratory, lowa State University, ${ }^{7}$ Department of Physics and Astronomy, \\ Ames Laboratory, lowa State University, ${ }^{8}$ Physics Department and Centre for the Physics of \\ Materials, McGill University, ${ }^{9}$ Department of Physics and Astronomy, Ames Laboratory, lowa State \\ University, ${ }^{10}$ Department of Physics and Astronomy, Ames Laboratory, lowa State University, \\ ${ }^{11}$ Department of Physics and Astronomy, Harvard University, ${ }^{12}$ Department of Physics and \\ Astronomy, Ames Laboratory, lowa State University \\ ${ }^{13}$ Department of Physics and Astronomy, Ames Laboratory, lowa State University \\ simonr@ameslab.gov
}

EuIn2As2 is theoretically predicted to order in a collinear antiferromagnetic structure that preserves inversion symmetry and induces an axion-insulator state. Combining results from neutron diffraction, symmetry analyses and density functional theory, we show that EuIn2As2 instead exhibits low-symmetry helical antiferromagnetic order which breaks inversion. However, the combination of a $180^{\circ}$ rotation and time-reversal symmetry (C2 x T=2') elements still makes the compound a stoichiometric magnetic topological-crystalline axion insulator. Surfaces normal to the 2' symmetry axes show exotic gapless Dirac cones (DC) which are unpinned to specific time-reversal invariant momenta (TRIM). Gapped DC pinned to TRIM appear on the other surfaces. Dissipationless charge transport arises on the 2' protected surfaces while half-integer quantum anomalous-type conductivity occurs on the other surfaces. We predict that modest values of applied magnetic field can switch between the different surfaces states. 